\title{
SINONASAL MUCOSAL MELANOMA: A CLINICOPATHOLOGICAL REVIEW
}

\author{
Soumyajit Das ${ }^{1}$, Subhasish Mukherjee ${ }^{2}$
}

${ }^{1}$ Assistant Professor, Department of ENT, Sikkim Manipal Institute of Medical Sciences. ${ }^{2}$ Associate Professor, Department of ENT, Sikkim Manipal Institute of Medical Sciences.

\begin{abstract}
Melanomas are malignant tumours, which originate from melanocytes. Melanocytes originate from the neural crest cells and then migrate to various tissues in the body during the course of development. Sinonasal melanoma are rare tumours and the aetiopathogenesis, treatment and staging of the tumours are still an area of debate. There remains paucity of studies dealing exclusively with sinonasal melanoma. We attempt to make a comprehensive study of the disease by reviewing the existing litera ture on sinonasal melanoma.
\end{abstract}

\section{METHODOLOGY}

Review of existing literature on the melanoma of nose and paranasal sinus.

\section{RESULTS AND DISCUSSION}

The melanoma of the sinonasal tract are rare and account for $1 \%$ of all melanoma. The aetiology of the tumour is still debated and the behaviour of the tumour remains conspicuously different both genetically and clinically from its cutaneous counterpart. Surgery remains the mainstay of treatment, though newer modalities are being explored. The prognosis of these tumours remain grim with a 5 -year survival rate of about $25 \%$.

\section{CONCLUSION}

Early diagnosis, histopathological confirmation and aggressive control of the primary tumour is the standard modality of treatment. The molecular biology and genetics of the tumour are an interesting field of research and targeted therapy may hold the key to improve the outcome in the future.

\section{KEYWORDS}

Melanoma, Mucosal, Nose and Paranasal Sinus, Staging, Immunohistochemistry, Treatment, Prognosis.

HOW TO CITE THIS ARTICLE: Das S, Mukherjee S. Sinonasal mucosal melanoma: a clinicopathological review. J. Evolution Med. Dent. Sci. 2016;5(42):2603-2606, DOI: 10.14260/jemds/2016/608

\section{INTRODUCTION}

Melanomas are primarily tumours arising from the melanocytes in the skin. Melanocytes originate from the neuroectoderm. However, rarely melanomas may also arise from the mucosal layer of the paranasal sinuses, gastrointestinal tract and the urogenital system. Though there has been an exponential increase in the incidence of cutaneous melanoma the incidence of mucosal melanomas have remained the same over the years. The prognosis of mucosal melanomas have also not changed much and the five-year survival rate remains grim. As the pathogenesis, staging, modality of treatment still remains unclear and the prognosis of the disease is poor, a review was carried out of the existing literature, recent trends and concepts in the management of these rare tumours, so as to bring forth a comprehensive study of the disease.

\section{METHODOLOGY}

A search of the English literature of last fifteen years was conducted in PubMed/Medline and Google Scholar using the

Financial or Other, Competing Interest: None.

Submission 08-04-2016, Peer Review 02-05-2016,

Acceptance 09-05-2016, Published 25-05-2016.

Corresponding Author:

Dr. Soumyajit Das,

Assistant Professor,

Department of ENT,

Sikkim Manipal Institute of Medical Sciences,

$5^{\text {th }}$ Mile, Tadong, Gangtok - 737102,

Sikkim.

E-mail: drsoumya_entamch@rediffmail.com DOI: $10.14260 /$ jemds $/ 2016 / 608$ keywords Melanoma, Mucosal, Nose and Paranasal Sinus, Staging, Immunohistochemistry, Treatment and Prognosis. Literature on cutaneous melanoma or mucosal melanoma of gastrointestinal, genitourinary tract were excluded from the review. Case reports dealing with mucosal melanoma other than the sinonasal melanoma were excluded from the review. The search yielded articles which were categorized as review articles, case reports and retrospective analysis of melanoma of nose and paranasal sinuses. Most studies were single centre studies with sample size ranging from ten cases to about 186 cases. A single study of sample size 815 based on national cancer registry was also retrieved for review.

\section{DISCUSSION}

Melanoma mostly arises from the skin. However, extracutaneous origin of melanomas is also known. Primary mucosal melanoma arises from various organs like the nose and paranasal sinuses, gastrointestinal tract and the urogenital system. Primary mucosal melanoma are rare and account for only $1.4 \%$ of all melanomas.[1] The first case of mucosal melanoma was described by Lincoln et al in 1885.[2] The most common site of mucosal melanoma is the head and neck region and 55\% of all mucosal melanoma occur in this region. ${ }^{[3]}$ Mucosal melanomas of the head and neck region comprise less than $1 \%$ of all melanomas.[4,5] The sites for head and neck mucosal melanoma are usually the nose, paranasal sinuses and the oral cavity. It is important to keep in mind metastasis from a cutaneous lesion, while establishing the diagnosis of primary mucosal melanoma. It is seen that metastasis from cutaneous melanoma may occur into the 
mucosa of the sinonasal tract rarely in less than $1 \%$ of the cases.[6]

Primary mucosal melanoma of the sinonasal tract arise de novo from the melanocytes in the surface epithelium or the stroma. Pre-existing nevus seem to have a little role in the pathogenesis of these tumours.[6] The aetiological agents responsible for mucosal melanoma are not established. Tobacco smoke and occupational exposure to formaldehyde have been implicated as possible aetiological factors. Thompson et al, in their retrospective analysis of 115 cases of sinonasal tract mucosal melanoma found that $7.5 \%$ of patients had an occupational exposure to formaldehyde.[6] The role of formaldehyde in the causation of sinonasal melanoma needs further investigation. Genetic studies have revealed that the mucosal melanomas carry different genetic variation than their cutaneous counterparts. Studies show that $75 \%$ of cutaneous melanoma arising from the sun exposed areas carry mutations in BRAF oncogene, which is rare in the mucosal variants (Approximately 2\%).[2,7]

Mucosal melanomas mostly carry C-KIT expression (88\%) with $5-20 \%$ of tumours carrying active C-KIT mutations. ${ }^{[2]} \mathrm{C}$ KIT is a key regulator involved in the proliferation, migration and differentiation of melanocytes. Zanoni et al, in their molecular analysis of sinonasal melanomas found that $48.1 \%$ of the tumours lost the tumour suppressor gene PTEN and in $55.2 \%$ of the tumours the p16 gene was absent.[8] The same study also noted that sinonasal melanomas carried an overexpression of CCND1 protein (65.6\%) and that significant tumour suppression was achieved by antagonizing CCND1 protein. The clinical significance of these genetic aberrations in terms of prognosis and outcome of the disease are not known at present, but may hold the key to newer therapeutic options in the future.

Mucosal melanoma usually presents after the $6^{\text {th }}$ decade. Thompson et al, in their retrospective analysis of 115 patients reported the youngest patient who was only 13 years of age.[6] Sinonasal mucosal melanoma have been shown to affect both sexes equally.[6,9] However, study on a large series of 186 patients of Sweden shows a significant female preponderance of the disease.[10] On the other hand, study by Mochel et al found a male-female ratio of 3:2 in their series of sinonasal melanoma.[11] Mendehall et al in their review also found a slight male preponderance of the disease. ${ }^{[4]}$ This discrepancy in the gender predisposition of the disease may be due to rare nature of the disease and that most of studies reported so far have been institutional studies with relatively small sample size. Mucosal melanomas have rich vascularisation and often arise from hidden areas of the head and neck region.

Unlike their cutaneous counterpart, mucosal melanomas present at an advanced stage and have poor outcome. Nose and the paranasal sinuses are the most common sites of mucosal melanoma of the head and neck region; $80 \%$ of these tumours arise from the nasal cavity and nearly $18 \%$ arise from the paranasal sinuses. The common subsites in the nasal cavity include septum (41\%), middle turbinate (29\%), inferior turbinate (23\%), and the lateral nasal wall (7\%).[3] The symptoms of these tumours are often nonspecific and may range from epistaxis, nasal obstruction, proptosis, diplopia, facial pain and asymmetry depending upon the site of origin and spread of the tumour. Compared to their oral counterpart sinonasal melanoma have lower rate of regional spread and more commonly present as ulcerative or polypoid morphology, pseudopapillary growth pattern with necrosis and a tendency towards perineural invasion. ${ }^{[12]}$

Mucosal melanoma may be suspected from their gross appearance presenting as a large polypoidal and bulky mass which may or may not contain melanin pigment. Microscopically, these tumours usually show lymphoid infiltrate at the periphery which may be due to immune response.[6]; $20-25 \%$ of the mucosal melanoma are amelanotic making diagnosis difficult at histopathology. It is interesting to note that Langard $\mathrm{M}$ et al in their study on 186 patients of sinonasal mucosal melanoma in the Swedish population found that about $70 \%$ of the tumours were amelanotic clinically. ${ }^{[10]}$ These amelanotic variant may be associated with a worse prognosis. Studies suggest that the presence of mitotic figure $(>10 / 10 \mathrm{HPF})$ may be associated with worse clinical outcome.[6,12]

The differential diagnosis of sinonasal mucosal melanoma may include undifferentiated carcinoma, lymphoma, plasmacytoma, rhabdomyosarcoma and olfactory neuroblastoma.[6] which may not be reliably differentiated on histology. Histopathological confirmation often requires immunohistochemical staining to differentiate melanoma from other lesions. Commonly used markers for mucosal melanoma include S-100, HMB 45, Melan A, tyrosinase and Microphthalmia Transcription Factor (MITF). S-100 is the most sensitive stain for melanocytes, but lacks specificity and stains approximately $95 \%-100 \%$ of the tumours when compared with HMB 45 and Melan A which stain approximately $86 \%$ and $84 \%$ of the tumours.[11,13] Moris et al evaluated the role of PNL2 melanocytic marker in immunohistochemical staining of mucosal melanomas.

They found that PNL2 stained the melanocytes diffusely in most of the tumours and that the intensity of the staining was stronger than HMB 45.[14] Aung P et also found PNL2 to be more specific and superior to other markers for diagnosing metastatic melanoma. Diagnosing the desmoplastic variant may be difficult and studies have shown that most of the melanoma marker including PNL2 fail to detect these rare variant.[15] A newer melanoma marker KBA62 was evaluated and found to be positive for the desmoplastic melanoma.[15] Literature also shows a case of sinonasal melanoma showing plasmacytoid variant with positive staining of CD 138, which is a plasma cell marker.[13] The prognostic significance of such aberrations are not known. However, such aberrant cases may pose a diagnostic challenge for the pathologist and the clinician if one is unaware of it.

The staging of mucosal melanoma has been an area of debate. The Clark and Breslow staging of cutaneous melanoma has no significance in assessing the prognosis of mucosal melanoma. This system is inapplicable in mucosal melanoma, because of lack of histological landmark of the papillary and reticular dermis and inability to assess the true depth of invasion of the tumour. The Ballantyne system of classification grouped mucosal melanoma into three stages, viz. Stage 1local disease, Stage II - Regional spread and Stage III - systemic metastasis. This staging system is simple and approximately $70-95 \%$ of mucosal melanoma are found to present as a Stage I localized disease.[12] However, such a large portion falling in one category limits the prognostic significance of the Ballantyne staging system.

Prasad et al proposed a microstaging system for localized node negative tumours based on the level of invasion to tissue 
depth. They found the staging to be an independent predictor of prognosis of the disease.[16] Thompson LD et al proposed a staging system after their clinicopathological analysis of 115 cases and found the system to provide "statistically meaningful predictive value" of the outcome of sinonasal mucosal melanoma.[6] This system requires further validation with a larger sample size. Currently, the consensus is on the use of AJCC staging system as the tumours are fairly distributed in various stages unlike the Ballantyne system and provides stage specific prognosis of the disease.[1,12]

The mainstay of treatment for sinonasal melanoma is surgery. The extent of surgical resection depends on the local spread of the tumour, which may often be limited by the closeness of the important structures. Histologically, tumour free margins of resection did not give statistically significant better survival rates in various studies.[12,17,18] Prophylactic neck dissection in absence of nodal disease is not recommended and the role of sentinel node biopsy is under study. ${ }^{[19]}$ Adjuvant radiotherapy has been shown to improve locoregional control. However, its effect on the long term survival is doubtful. ${ }^{[7]}$ Conventional radiotherapy as a primary modality of treatment has been shown to achieve a 5-year local control rate of $13 \%$, while postoperative radiotherapy achieves a rate of $30 \% .{ }^{[20]}$ Liao JJ et al studied the effects of fast neutron radiotherapy on primary mucosal melanoma and found an overall local control rate of $79 \% .{ }^{[20]}$

Other modalities of radiotherapy like intensity modulated radiotherapy, proton therapy and carbon ion therapy have shown promising results in clinical trials. ${ }^{[7]}$ Further validation with a large sample size is required to assess the effectiveness of these modalities. Chemotherapy has been mostly used for palliation and dacarbazine has been used as the standard chemotherapeutic agent. However, studies are underway to assess the efficacy of targeted therapy and immunotherapy. Clinical trials with Vemurafenib (A selective BRAF inhibitor) and Imatinib (Therapeutic agent targeting C-KIT mutation) have shown promising results.[7] Immunobiological therapy involving transfer of Lymphokine Activated Killer (LAK) cells has shown to increase the 5-year survival rate in patients. ${ }^{[7,21]}$ These newer modalities of treatment provides an interesting field for further research and may open up newer horizons in the treatment of this rare disease.

The rare occurrence of mucosal melanoma is a limiting factor for analysis of the prognostic factors of the tumour. To date most of the data are derived from institutional studies. Jethanemest D et al in their study of 815 cases found age more than 70 years to be an independent risk factor and was associated with poor outcome of the disease. Primary tumour site was also found to effect the survival rate and it was found that in the nose and paranasal region, tumours arising in the nose had significantly better outcome than those arising from the paranasal sinus and nasopharynx.[22] This can be attributed to the late presentation of the paranasal and nasopharyngeal melanomas, which tend to remain occult. Apart from these, tumour size, nodal status and distant metastasis adversely affected the long-term survival. The overall 5-year and 10-year survival of sinonasal mucosal melanoma has been found to be $25.2 \%$ and $12.2 \%$ respectively.[22] The overall prognosis thus remain grim and most of the patients succumb to distant metastasis in spite of achieving a clear surgical margins of resection.[23,24]

\section{CONCLUSION}

Mucosal melanoma of the nose and paranasal sinus are rare tumours with an adverse clinical outcome. The prognosis of these tumours have not improved significantly over the past. Early diagnosis and histopathological confirmation and aggressive control of the primary tumour is the standard care. The molecular biology and the genetics of the tumour are an interesting field of research and targeted therapy may hold the key to improve the outcome in the future. The literature pertaining to the subject has been mostly retrospective analysis of data in institutional setup and the current consensus is derived from these institutional data. Studies in India are limited. The geographical and racial factors and the genetics of the tumour can only be evaluated from larger studies. This can be overcome by institutional collaboration and undertaking multicentric studies, which may be helpful in formulation of specific guidelines for the treatment of these tumours.

\section{REFERENCES}

1. Mihajlovic M, Vlajakovic S, Jovanovic P, et al. Primary mucosal melanomas: a comprehensive review. Int J Clin Exp Pathol 2012;5(8):739-53.

2. Seetharamu N, Ott PA, Pavlick AC. Mucosal melanomas: a case-based review of the literature. The Oncologist 2010;15(7):772-81.

3. Molina JPD, Tapia JPR, Pendas JLL, et al. Sinonasal mucosal melanomas. Review of 17 cases. Acta Otorrinolaringol Esp 2008;59(10):489-93.

4. Mendenhall W, Amdur RJ, Hinerman RW, et al. Head and neck mucosal melanoma. Am J Clin Oncol 2005;28(6):626-30.

5. Liétin B, Montalban A, Louvrier C, et al. Sinonasal mucosal melanomas. European Annals of Otorhinolaryngology, Head and Neck diseases 2010;127(2):70-6.

6. Thompson LD, Wieneke JA, Miettinen M. Sinonasal tract and nasopharyngeal melanomas a clinicopathologic study of 115 cases with a proposed staging system. The American Journal of Surgical Pathology 2003;27(5):594611.

7. Li W, Yu Y, Wang H, et al. Evaluation of the prognostic impact of postoperative adjuvant radiotherapy on head and neck mucosal melanoma: a meta-analysis. BMC Cancer 2015;15:758. DOI 10.1186/s12885-015-1750-7.

8. Zanoni MT, Medicina D, Lombardi D, et al. Sinonasal mucosal melanoma: molecular profile and therapeutic implications from a series of 32 cases. Head Neck 2013;35(8):1066-77. doi: 10.1002/hed.23079.

9. Prasad ML, Busam KJ, Patel SG, et al. Clinicopathologic differences in malignant melanoma arising in oral squamous and sinonasal respiratory mucosa of the upper aerodigestive tract. Arch Pathol Lab Med 2003;127(8):997-1002.

10. Jangard M, Hansson J, Ragnarsson-Olding B. Primary sinonasal malignant melanoma: a nationwide study of the Swedish population, 1960-2000. Rhinology 2013;51(1):22-30.

11. Mochel MC, Duncan LM, Piris A, et al. Primary mucosal melanoma of the sinonasal tract: a clinicopathologic and immunohistochemical study of thirty-two cases. Head and Neck Pathol 2015;9(2):236-43. DOI 10.1007/s12105-014-0570-4. 
12. Moreno MA, Roberts DB, Kupferman ME, et al. Mucosal melanoma of the nose and paranasal sinuses, a contemporary experience from the MD. Anderson cancer center. Cancer 2010;116(9):2215-23. doi: 10.1002/cncr.24976.

13. Chirakalwasan N. Primary sinonasal mucosal melanoma with CD138 expressed plasmacytoid cells: a case report and review of literature. Asian Archives of Pathology 2013;9(2):72-8.

14. Morris LG, Wen YH, Nonaka D, et al. PNL2 melanocytic marker in immunohistochemical evaluation of primary mucosal melanoma of the head and neck. Head Neck 2008;30(6):771-5. doi: 10.1002/hed.20785.

15. Aung P, Rikala MS, Lasota J, et al. KBA62 and PNL2: two newer melanoma markers-immunohisto-chemical analysis of 1563 tumours including metastatic, desmoplastic, and mucosal melanomas and their mimics. Am J Surg Pathol 2012;36(2):265-72. doi: 10.1097/PAS.0b01 3e31823651cb.

16. Prasad ML, Patel SG, Huvos AG, et al. Primary mucosal melanoma of the head and neck: a proposal for microstaging localized, Stage I (lymph node-negative) tumours. Cancer 2004;100(8):1657-64.

17. Bachar G, Loh KS, O'Sullivan B, et al. Mucosal melanomas of the head and neck: the princess margaret hospital experience. Head Neck 2008;30(10):1325-31.
18. Gupta T, Agarwal JP, Singh S, et al. Mucosal melanoma of the head and neck: tata memorial experience. International Journal of Head and Surgery 2010:1(3):141-5.

19. Gilain L, Houette A, Montalban A, et al. Mucosal melanoma of the nasal cavity and paranasal sinuses. European Annals of Otorhinolaryngology, Head and Neck diseases 2014;131(6):365-9.

20. Liao JJ, Parvathaneni U, Laramore GE, et al. Fast neutron radiotherapy for primary mucosal melanomas of the head and neck. Head Neck 2014;36(8):1162-7. doi: 10.1002/hed.23428.

21. Kanetaka S, Tsukuda M, Takahashi M, et al. Mucosal melanoma of the head and neck. Experimental and Therapeutic Medicine 2011;2(5):907-10.

22. Jethanamest D, Vila PM, Sikora AG, et al. Predictors of survival in mucosal melanoma of the head and neck. Ann Surg Oncol 2011;18(10):2748-56. doi:10.1245/s10434011-1685-4.

23. Breik O, Sim F, Wong T, et al. Survival outcomes of mucosal melanoma in the head and neck: case series and review of current treatment guidelines. J Oral Maxillofac Surg 2016;S0278-2391(16)00342-6.

24. Samstein RM, Carvajal RD, Postow MA, et al. Localized sinonasal mucosal melanoma: outcomes and associations with stage, radiotherapy, and positron emission tomography response. Head Neck 2016. doi: 10.1002/hed.24435. 